\title{
Weighted gene co-expression network analysis identifies key genes from extracellular vesicles as potential prognostic biomarkers for congenital pulmonary stenosis
}

\author{
ZIRUI HUANG ${ }^{1-3^{*}}$, XIAOHONG LI $^{1 *}$, MIN QIU $^{2}$, JING CHEN $^{1}$, MIAO TIAN $^{2,3}$, FENGZHEN HAN $^{4}$, \\ YANQIU OU ${ }^{5}$, XIAOQING LIU ${ }^{5}$, CHENGBIN ZHOU ${ }^{1}$, HAIYUN YUAN $^{1}$, JIAN ZHUANG $^{1,2}$ and JIMEI CHEN ${ }^{1,2}$ \\ ${ }^{1}$ Guangdong Provincial Key Laboratory of South China Structural Heart Disease, Guangdong Cardiovascular Institute; \\ ${ }^{2}$ School of Medicine, South China University of Technology School of Medicine; \\ Departments of ${ }^{3}$ Cardiovascular Surgery, ${ }^{4}$ Obstetrics and Gynecology and ${ }^{5}$ Epidemiology, Guangdong Cardiovascular \\ Institute, Guangdong Provincial People's Hospital, Guangdong Academy of Medical Sciences, \\ Guangzhou, Guangdong 510080, P.R. China
}

Received February 15, 2020; Accepted May 27, 2020

DOI: $10.3892 / \mathrm{mmr} .2020 .11332$

\begin{abstract}
Pulmonary stenosis (PS) is a congenital heart disease characterized by a dynamic or fixed anatomic obstruction of blood flow from the right ventricle to the pulmonary arterial vasculature. In the present study, extracellular vesicle long RNAs (EVLRs) from pregnant females who had healthy infants or PS infants were analyzed by RNA sequencing, and their diagnostic potential for PS during pregnancy was evaluated. A method for the selection of genes that could be considered as informative for the prediction PS based on extracellular vesicles (EVs) from pregnant females using long-read RNA sequencing was developed. Blood samples were collected from females carrying fetuses with PS and females carrying unaffected fetuses ( $\mathrm{n}=6$ in each group). Physical characterization of EVs was performed using nanoparticle tracking analysis, transmission electron microscopy and western blotting. EVLRs from plasma were profiled by RNA sequencing and mRNA co-expression modules were constructed by weighted gene co-expression network analysis (WGCNA). Gene Ontology (GO) enrichment analysis was used to predict the function of the genes in each module. Hub genes were filtered out based on WGCNA and visualized using Cytoscape. EVLRs
\end{abstract}

Correspondence to: Professor Jian Zhuang or Professor Jimei Chen, Guangdong Provincial Key Laboratory of South China Structural Heart Disease, Guangdong Cardiovascular Institute, 106 Zhongshan Second Road, Yuexiu, Guangzhou, Guangdong 510080, P.R. China E-mail: drzhaungjian5413@163.com

E-mail: jimei1965@gmail.com

\section{"Contributed equally}

Key words: pulmonary stenosis, extracellular vesicle, co-expression module, biomarker consisted of mRNAs, microRNAs and long non-coding RNA. Overall, 26 modules were identified containing 16,394 genes. All modules were independent of each other. One particular module, referred to as the blue module, was markedly different between the two groups. A total of 735 hub genes in the blue module were identified, of which 33 were visualized, demonstrating the connection between these hub genes. GO enrichment analysis demonstrated that the analyzed hub genes were enriched in 'glucose transport', 'ATP-dependent chromatin remodeling', 'histone deacetylation', 'histone H3-K4 methylation', 'DNA methylation', 'apoptotic signaling pathway' and 'glucocorticoid receptor signaling pathway'. The hub genes identified in this module may provide a genetic framework for prenatal PS diagnosis. Furthermore, functional analysis of these associated genes may provide a theoretical basis for further research on PS pathogenesis.

\section{Introduction}

Pulmonary stenosis (PS) is a congenital heart disease (CHD) caused by abnormal development of the fetal heart during the first eight weeks of pregnancy, accounting for $\sim 6.2 \%$ of all CHD cases worldwide (1). PS can be classified into valvular, subvalvular and supravalvular subtypes (2). The symptoms of PS can be mild, moderate or severe (3), and patients with severe PS require therapy due to possibly life-threatening complications. The etiology and pathogenesis of PS remain poorly understood (2). Current diagnostic methods for PS include electrocardiography, echocardiography, magnetic resonance imaging, multislice computed tomography, cardiac catheterization and angiography (2). However, biomarkers for early prediction and diagnosis remain unavailable. Several risk factors are associated with the onset of CHD, including genetic, epigenetic and environmental factors $(4,5)$, and this may also be the case for PS. However, to the best of the authors' knowledge, no genetic assessment for fetal PS during pregnancy has been conducted. 
Extracellular vesicles (EVs) are secreted as exosomes after fusion of endosomes with the plasma membrane or shed from the plasma membrane as microvesicles (6). EVs are involved in cell-to-cell communication between tumor cells and surrounding cells in the primary tumor microenvironment (7). Moreover, previous studies indicated that EVs play an important role in the maternal system as part of the physiological changes taking place during pregnancy. Cell-free DNA fragments released from the fetus are found in maternal circulation, and EVs can also be released from the fetus, placenta and maternal body into fetal-maternal circulation (8). EVs are detectable in maternal circulation as early as the sixth week of pregnancy (8). The concentration of EVs in circulation is higher in pregnant females compared with non-pregnant females and increases with severity of pregnancy-related disease, such as gestational diabetes mellitus, fetal growth restriction and preterm birth, and oxidative stress (9).

Various functional proteins, RNAs, lipids and metabolites are detectable in different classes of EVs (6). Since microenvironmental parameters such as acidosis, hypoxia and elevated interstitial fluid pressure can influence the release of EVs, optimized in vitro models that mimic in vivo cellular environments are required for the study of EVs $(10,11)$. EVs can contain several types of RNA, including mRNA, circular RNA (circRNA) and long non-coding RNA (lncRNA) (12). Functional extracellular vesicle long RNAs (EVLRs) found in peripheral blood could represent biomarkers for the diagnosis of a variety of diseases, including hepatic tumors, gastric cancer, kidney cancer and breast cancer $(13,14)$. However, the characteristics of EVLRs in pregnant females with PS infants remain largely unknown. Therefore, understanding the roles of plasma EVLRs in pregnant females may provide new predictive strategies for the diagnosis of fetal PS in pregnant females.

In the present study, EVLRs from pregnant females who had healthy infants or infants with PS were analyzed by RNA sequencing and evaluated for their potential use as diagnostic markers for PS during pregnancy. The present study provides a comprehensive analysis of hub genes associated with EVLRs. The identification of these hub genes may provide insight into improved diagnostic approaches for PS during pregnancy.

\section{Materials and methods}

Sample collection. Peripheral plasma samples from pregnant females were provided by Guangdong Provincial People's Hospital for high-throughput sequencing. All subjects gave their verbal informed consent before enrollment. The study was conducted in accordance with the Declaration of Helsinki, and the protocol was approved by the Ethics Committee of Guangdong Provincial People's Hospital. Blood samples were obtained between September 2017 and November 2018 from pregnant females with children affected by PS, as well as pregnant females carrying unaffected fetuses ( $\mathrm{n}=6$ in each group). All blood samples were drawn 1-2 days before delivery. PS was diagnosed using a postnatal cardiac color ultrasound. Both groups consisted of three male infants and three female infants. The mean age of pregnant females in the PS and control groups was 33.3 and 29 years, respectively.
Isolation of EVs. Plasma EVs were extracted using an exoRNeasy Serum/Plasma Midi kit (Qiagen $\mathrm{GmbH}$ ) and $\sim 1 \mathrm{ml}$ plasma was centrifuged at $16,000 \mathrm{xg}$ for $10 \mathrm{~min}$ at $4^{\circ} \mathrm{C}$. Cellular materials and coagulated proteins were removed, and the supernatant was transferred into a new tube by careful aspiration. The re-suspended EV liquid was subsequently used for characterization of EVs.

Nanoparticle tracking analysis. The size distribution of the isolated EVs was analyzed using NanoSight NS300 (Malvern Panalytical). Particles were automatically tracked and sized based on Brownian motion and the diffusion coefficient. After isolation, EVs were diluted in $1 \mathrm{ml}$ of exosome-free PBS, and the mixture was slowly injected into a clean particle-free sample pool to avoid formation of bubbles. The sample pool was covered and placed into the instrument. Manipulations were performed according to the manufacturer's instructions. Three recordings were carried out for each sample and results are presented as an average of these recordings.

Transmission electron microscopy (TEM). For TEM, $3 \mu 1$ of EV pellet were placed on 200-mesh EM copper grids for $5 \mathrm{~min}$, incubated for $5 \mathrm{~min}$ at room temperature, and then subjected to standard uranyl acetate staining at room temperature for 1-2 min. The grids were then washed three times with PBS and allowed to semi-dry at room temperature. Subsequently, the grids were visualized with a transmission electron microscope (H7650; Hitachi, Ltd.) at magnification, x300, using digital micrograph software (v3.8; Gatan, Inc.).

Western blotting. EV lysate supernatants were prepared, total protein was extracted using Exosome cracking solution (Shanghai Umibio Biotechnology), and protein concentrations were determined using a bicinchoninic acid protein assay kit (Thermo Fisher Scientific, Inc.). Proteins $(60 \mu \mathrm{l})$ were separated via $10 \%$ SDS-PAGE, and subsequently transferred onto PVDF membranes (EMD Millipore). Then, 5\% non-fat milk in TBS with $0.05 \%$ Tween-20 (TBST) buffer was used to block the membranes for $1 \mathrm{~h}$ at room temperature. The membranes were incubated with rabbit anti-CD63 (1:500; cat. no. sc-5275; Santa Cruz Biotechnology, Inc.), anti-CD9 (1:1,000; cat. no. ab263019; Abcam) and anti-calnexin antibodies (1:1,000; cat. no. ab22595; Abcam) at $4^{\circ} \mathrm{C}$ overnight, washed with TBST, then incubated with horseradish peroxidase-conjugated goat anti-rabbit secondary antibody (1:5,000; cat. no. 7074S; Cell Signaling Technology, Inc.). Protein bands were visualized with an ECL reagent (Merck KGaA) using an automatic imager (GE Healthcare).

Long RNA sequencing and data processing. Long RNAs from EVs were amplified by Epi ${ }^{\mathrm{TM}}$ longRNA Ampli kit (Epibiotek). RNA sequencing libraries were constructed using SMARTer Stranded Total RNA-Seq kit (cat. no. 634413; Clontech Laboratories, Inc.). Adapters and low-quality bases were removed using Trimmomatic software (v0.36) (15). Clean RNA sequencing data were aligned to the GRCh38 human genome (hg38) downloaded from Ensembl (www.ensembl.org) using HISAT2 software (v2.1.0) (16). Genes were annotated using GENCODE annotation (v25; www.gencodegenes.org), and the read count for each gene was obtained using featu- 
A

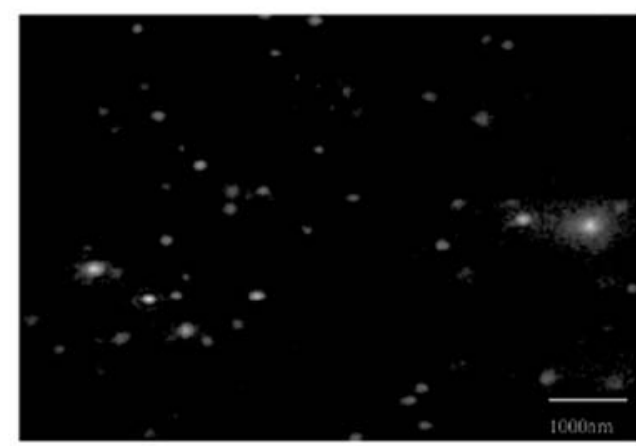

C

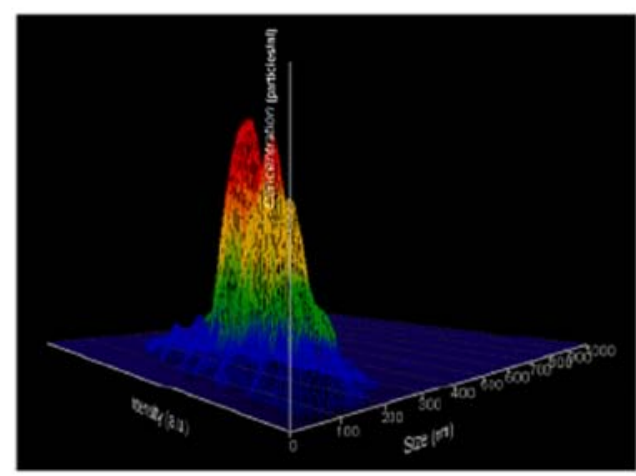

$\mathrm{E}$

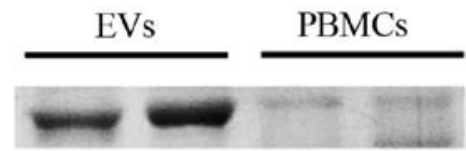

CD63

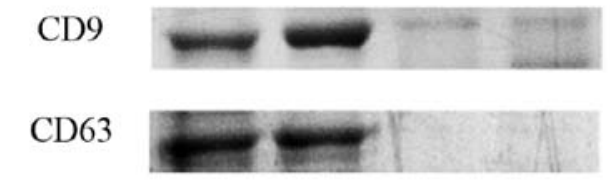

Calnexin
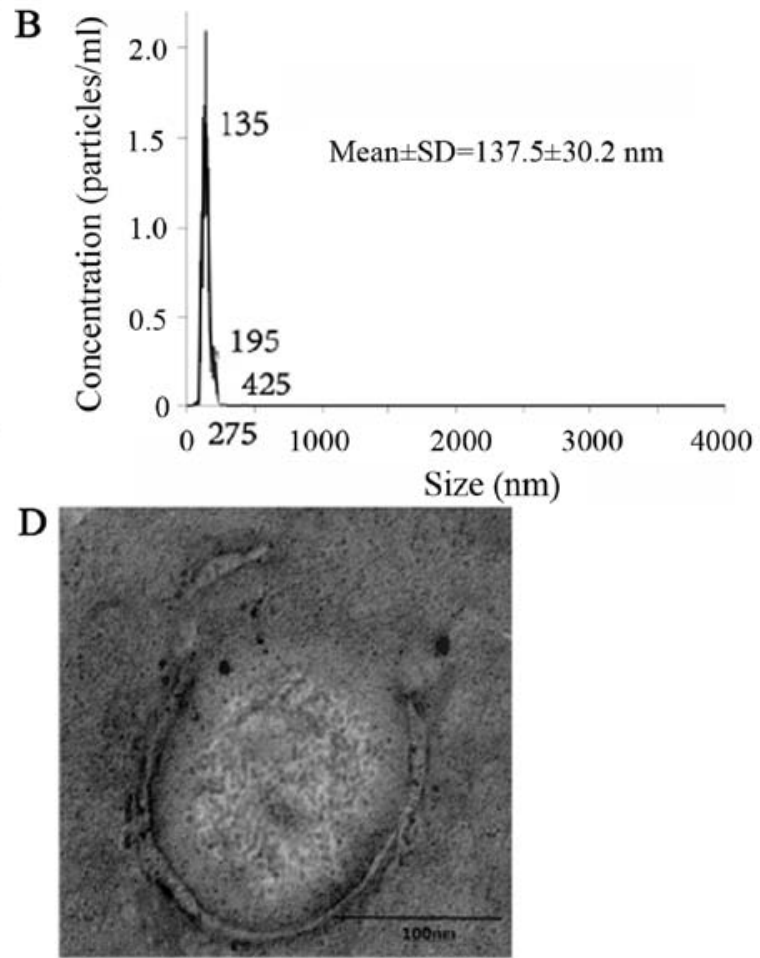

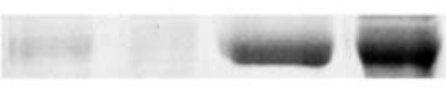

Figure 1. Characteristics of EVs in peripheral blood samples. (A) A representative screenshot of the NTA videos. Bright white dots indicate a moving particle. (B) NTA-estimated size distribution of isolated EVs. (C) Heat map pattern of EVs. (D) Representative transmission electron microscopy image of EVs. Scale bar, $100 \mathrm{~nm}$. (E) Western blot analysis of unenriched peripheral blood mononuclear cells and exosome-enriched vesicles. EV, extracellular vesicle; NTA, nanoparticle trafficking analysis; PBMC, peripheral blood mononuclear cells; SD, standard deviation; a.u., absorbance unit.

reCounts (v1.6.3; subread.sourceforge.net). Clean read counts were further normalized using the fragments per kilobase per million mapped reads method. Moreover, underexpressed genes in any sample were filtered out. Cluster analysis was performed using the flashClust R (v1.01-2-2) package (17).

Construction of co-expression modules of CHD. In weighted gene co-expression network analysis (WGCNA), the connectivity between two genes is the $\beta$ power of their correlation coefficient. The $\beta$ value determined both the scale-free topology fitting index and the mean connectivity of genes. The $\beta$ value for scale-free topology fitting index was $\geq 0.8$ by plotting the index against soft thresholds, which indicated that the topology of the network was scale-free, or independent, and simultaneously, the mean connectivity of genes was as high as possible, and it may result in several modules in the subsequent analysis for an insignificant mean connectivity. To understand relationship between them, the softConnectivity function from WGCNA package (v1.63) (18) was used, and the number of randomly selected genes was set to 5,000, while other parameters were set as default. The power was calculated by the pickSoftThreshold function in the WGCNA package. The expression values were compiled using the collapseRows function in the WGCNA package. Cluster analysis was subsequently performed using the flashClust function.

Detection of hub genes and functional enrichment analysis. Hub genes are defined as genes with high correlation in candidate modules. In the present study, genes in the same co-expression modules with module membership $\geq 0.8$ were considered as hub genes. Gene Ontology (GO; www. geneontology.org) enrichment analysis was carried out for each module and the corresponding gene data were mapped to the Database for Annotation, Visualization, and Integrated Discovery (DAVID; david.ncifcrf.gov/summary. jsp). A corrected P-value $<0.05$ was used as threshold. The most favorable module in the current study was visualized by Cytoscape v3.6.6 software (www.cytoscape.org), and the maximum intramodular connectivity of genes was considered as intramodular hub genes. 
A

Reads distribution

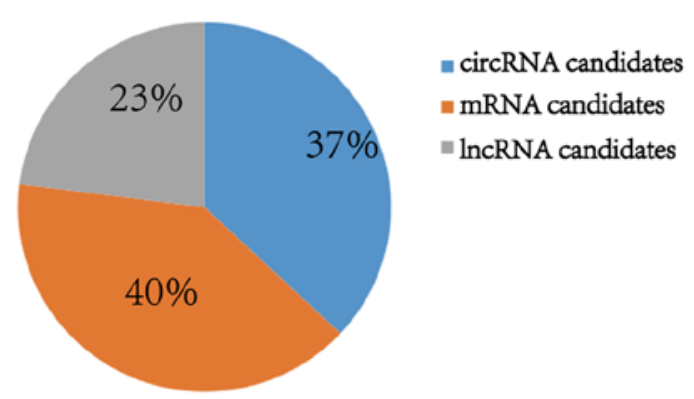

$\mathrm{B}$

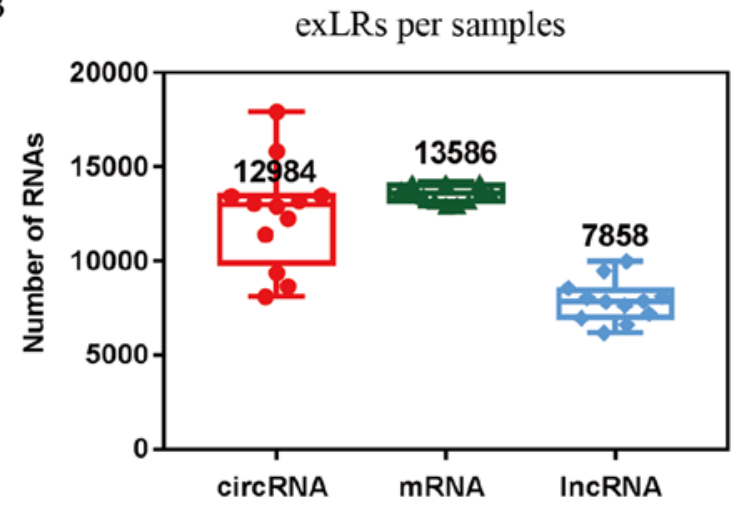

Figure 2. Analysis of samples by EVLR sequencing. (A) Distribution of annotated genes and identified circRNAs. (B) Number of EVLR in each sample. Data are presented as the median and the interquartile range. EVLR, extracellular vesicle long RNA; circRNA, circular RNA; lncRNA, long non-coding RNA.

A
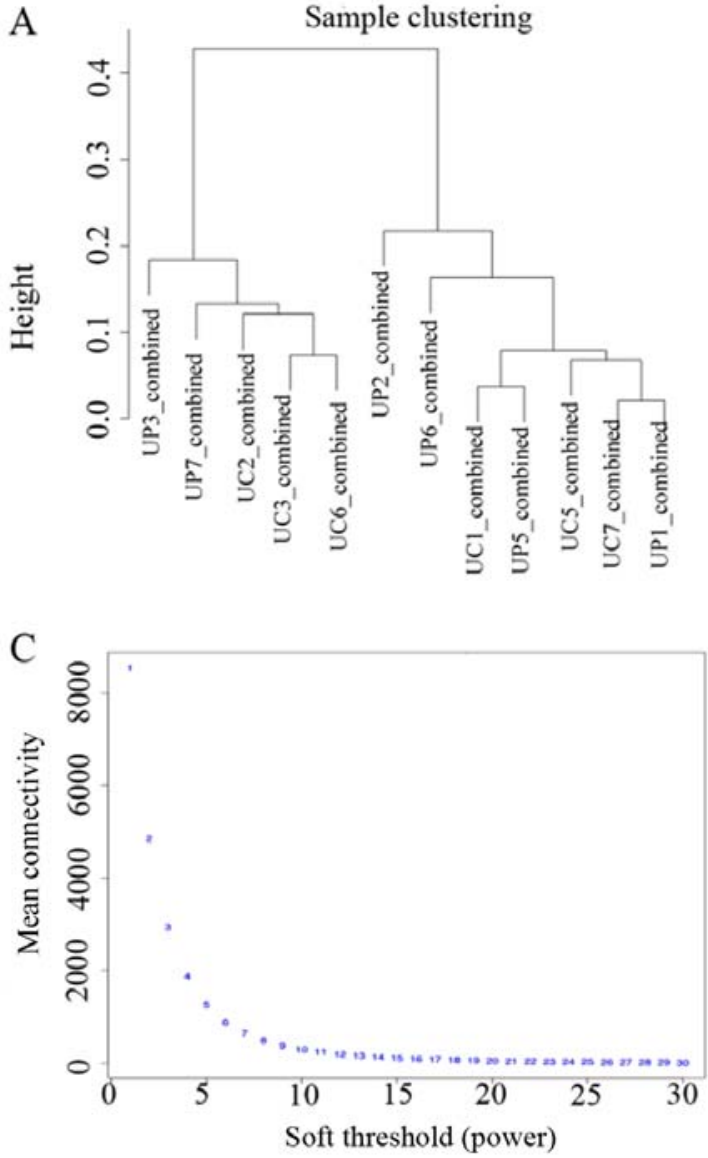

B
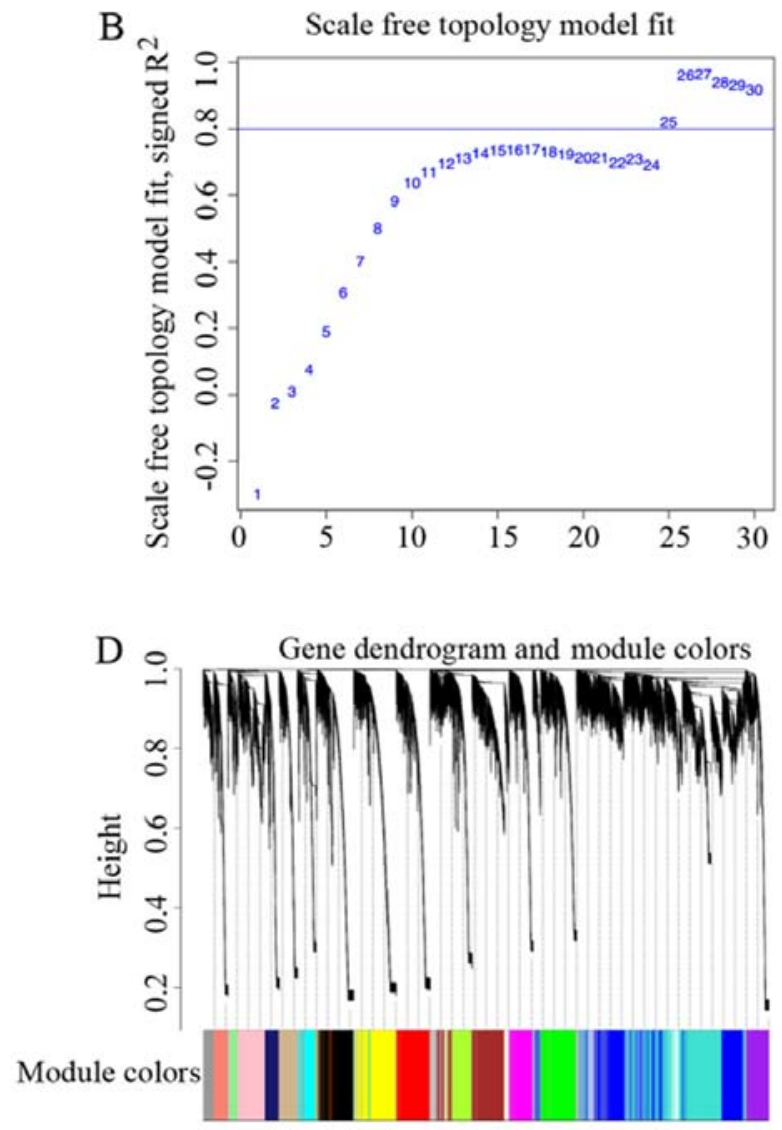

Figure 3. Co-expression module construction. (A) Cluster analysis of 12 samples based on gene expression. (B) Influence of soft-threshold power on scale-free topology fitting index. The blue numbers correspond with their abscissa and the blue line is the threshold of power value. (C) Influence of soft-threshold power on the mean connectivity. (D) Gene clustering dendrogram, with dissimilarity based on topological overlap, together with assigned color modules. A total of 26 co-expression modules were constructed and were displayed in different colors. UC, samples from the normal group; UP, samples from the PS group.

\section{Results}

Isolationand characterization of EVs.EVswere isolated and characterized morphologically and phenotypically. Measurements demonstrated that the concentration of the isolated particles was $9.55 \times 10^{9} \pm 3.27 \times 10^{7}$ particles $/ \mathrm{ml}$ (data not shown), and the mean diameter of the particles was $137.8 \pm 30.2 \mathrm{~nm}$ (Fig. 1A-C). EVs were round-shaped and membrane-enclosed (Fig. 1D). The exosomal markers CD63 and CD9 were detected in the isolated vesicles, but not in peripheral blood mononuclear cells, whereas the expression of calnexin, the marker of peripheral blood mononuclear cells, showed the opposite results (Fig. 1E).

EVLR-sequencing. EVLR-sequencing yielded a median read count of 27.98 million mapped reads/sample. Overall, mRNA constituted $40 \%$ of total mapped reads. Other types of RNA included $37 \%$ circRNAs and $23 \%$ lncRNAs (Fig. 2A). On average, 13,586 mRNAs, 12,984 circRNAs, and 
Table I. Number of genes in the 26 modules.

Module colors

Frequency

\begin{tabular}{lr}
\hline Black & 808 \\
Blue & 1,909 \\
Brown & 1,187 \\
Cyan & 407 \\
Dark green & 68 \\
Dark grey & 56 \\
Dark brown & 124 \\
Dark turquoise & 61 \\
Green & 997 \\
Green-yellow & 606 \\
Grey & 1,153 \\
Grey 60 & 284 \\
Light cyan & 404 \\
Light green & 246 \\
Light yellow & 208 \\
Magenta & 648 \\
Midnight blue & 54 \\
Orange & 781 \\
Pink & 640 \\
Purple & 943 \\
Red & 176 \\
Royal blue & 411 \\
Salmon & 515 \\
Tan & 2,242 \\
Turquoise & 1,062 \\
Yellow & \\
\hline &
\end{tabular}

7,858 lncRNAs were detected from the 12 samples (Fig. 2B). In total, 19,391 genes were detected by EVLR-sequencing in the 12 samples. Moreover, 2,997 genes were filtered out as their expression was too low in any sample. Thus, expression levels of 16,394 genes in 12 samples were used to construct the co-expression network by the WGCNA package, of these $40 \%$ were associated with mRNA, $37 \%$ with circRNA and $23 \%$ with IncRNA.

Construction of co-expression modules. Cluster analysis was conducted on the mRNAs detected in all 12 samples, using the flashClust package (Fig. 3A). Hub genes are displayed in gene dendrograms, in which they positively correlated together in different samples. Different soft-thresholding power values were analyzed. When the power value reached 25 , the scale-free topology fitting index was $\geq 0.08$ (Fig. 3B), and as the power value increased, the connectivity declined (Fig. 3C). Therefore, the power value used to construct co-expression module was 25 . A total of 26 distinct gene co-expression modules were constructed altogether (Fig. 3D). The number of genes in each module is listed in Table I.

Analysis of co-expression modules. As shown on the network heatmap plot (Fig. 4A), each module showed an independent expression to each other. Hence, we calculated eigengenes of each module, so as to quantify their co-expression similarity. Modules were clustered on the basis of their correlation with each other, which was consistent with the heatmap plot of the adjacencies (Fig. 4B).

Functional enrichment analysis and module visualization. Results of GO enrichment analysis are summarized in Table II. Biological process involved in fetal cardiovascular malformations, such as regulation of glucose transport and apoptotic signaling pathway were included in the blue module. Furthermore, the expression of each module in all samples was analyzed. Among all the 26 modules, the blue module markedly differed between the two groups (Fig. 5A). Additionally, a total of 735 genes were involved in the blue module. These genes were enriched in 'regulation of glucose transport', 'glucose transport', 'glucocorticoid receptor signaling pathway', 'ATP-dependent chromatin remodeling', 'histone deacetylation', 'histone H3-K4 methylation', 'DNA methylation' and 'apoptotic signaling pathways'. These biological processes were not enriched in other modules. The top 33 hub genes in the blue module, which have a high degree of correlation, are represented in an interaction network using Cytoscape software (Fig. 5B).

\section{Discussion}

WGCNA is a method used to investigate the relationship between gene expression and phenotype (18). In particular, WGCNA transforms gene expression data into co-expression modules, providing insight into signaling networks that may be responsible for phenotypic traits of interest (19). Not only can it illustrate how modules differ between control and experimental groups, it can also be used to examine the functions of genes within a particular module. WGCNA has been applied in the context of several diseases, such as osteosarcoma, glioma and renal cell carcinoma (20-23). In the present study, 26 co-expression modules were extracted from 16,394 genes using this method. Among these, one particular module, referred to as the blue module, displayed marked dissimilarity between PS group and normal group. The blue module was enriched in a variety of biological processes, which may influence fetal heart development, including 'glucose transport', 'ATP-dependent chromatin remodeling', 'histone deacetylation', 'histone H3-K4 methylation', 'DNA methylation', 'apoptotic signaling pathways' and 'glucocorticoid receptor signaling pathway'.

Maternal glycemia is a risk factor for the development of CHD in the fetus (24-27). A previous study indicated that maternal blood glucose levels were strongly associated with odds of tetralogy of Fallot (28). This highlights the need for further epidemiological and mechanistic investigation into the risk conferred by insulin signaling and glucose metabolism during early pregnancy. In the present research, genes of glucose transport in PS group were markedly downregulated compared with those in normal group.

A previous study suggested that maternal epigenetic changes were related to fetal cardiovascular malformations (29). Another study used genome-wide DNA methylation assays on placental samples and identified a 
Table II. GO enrichment analysis in blue, light cyan and grey 60 co-expression modules.

\begin{tabular}{|c|c|c|c|c|c|}
\hline Module & GO ID & GO Term & Difgene & GeneInGO & P-value \\
\hline \multirow[t]{8}{*}{ Blue } & GO:0010827 & regulation of glucose transport & 10 & 31 & $1.76 \times 10^{-7}$ \\
\hline & GO:0043044 & ATP-dependent chromatin remodeling & 8 & 23 & $1.61 \times 10^{-6}$ \\
\hline & GO:0016575 & histone deacetylation & 8 & 34 & $4.06 \times 10^{-5}$ \\
\hline & GO:0042921 & glucocorticoid receptor signaling pathway & 4 & 8 & $1.47 \times 10^{-4}$ \\
\hline & GO:0051568 & histone $\mathrm{H} 3-\mathrm{K} 4$ methylation & 6 & 23 & $2.07 \times 10^{-4}$ \\
\hline & GO:0015758 & glucose transport & 10 & 65 & $2.12 \times 10^{-4}$ \\
\hline & GO:0006306 & DNA methylation & 6 & 26 & $4.28 \times 10^{-4}$ \\
\hline & GO:0097190 & apoptotic signaling pathway & 13 & 130 & $1.86 \times 10^{-3}$ \\
\hline \multirow[t]{2}{*}{ Light cyan } & GO:0006413 & translational initiation & 53 & 157 & $2.18 \times 10^{-67}$ \\
\hline & GO:0006415 & translational termination & 42 & 89 & $9.93 \times 10^{-61}$ \\
\hline \multirow[t]{2}{*}{ Grey 60} & GO:0030097 & Hemopoiesis & 7 & 85 & $1.30 \times 10^{-6}$ \\
\hline & GO:0048821 & erythrocyte development & 7 & 19 & $6.02 \times 10^{-6}$ \\
\hline
\end{tabular}

GO, Gene Ontology; Difgene, genes in a certain module related to one specific biological process; GeneInGO, genes in GO dataset related to one specific biological process.

A

TOMplot (network heatmap plot)
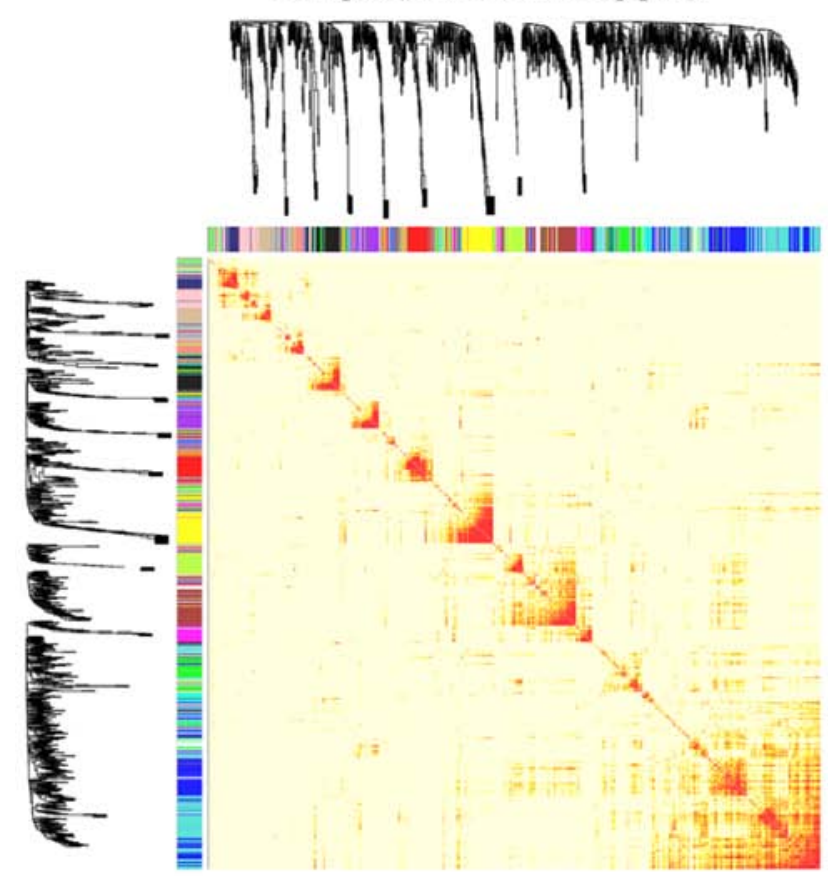

B
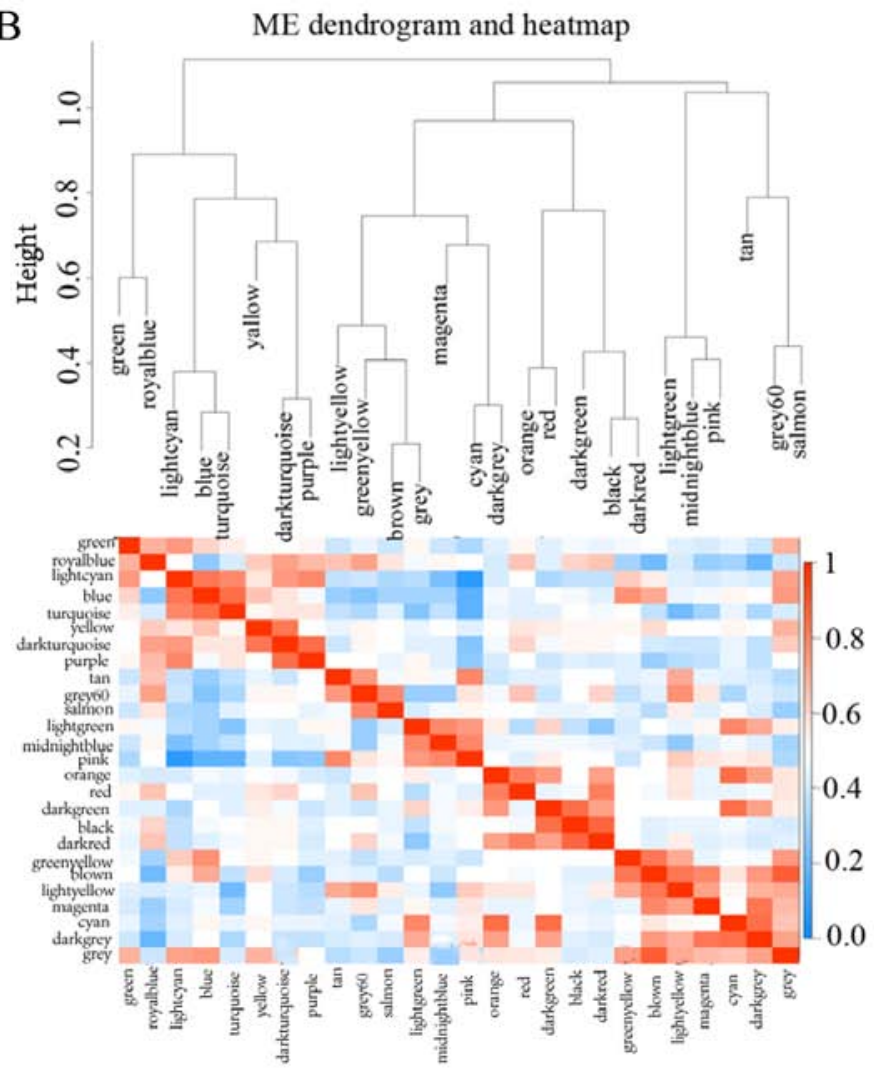

Figure 4. Network heatmap plot of co-expression modules. (A) Clustering dendrogram of genes and assignment of modules along the left side and the top. Each point in the figure represents the correlation between its corresponding modules on the horizontal axis and vertical axis. Followed by yellow color, the red color indicates the highest co-expression interconnectedness. Blocks of darker colors along the diagonal represent the modules. (B) Dendrogram of modules and heatmap based on their correlation with each other. Each cell in the figure represents the correlation between its corresponding modules on the horizontal axis and vertical axis. Red represents high adjacency (positive correlation) and blue shows low adjacency (negative correlation).

total of 80 highly accurate potential $\mathrm{CpG}$ sites for detection of ventricular septal defects (30). These differentially methylated genes were previously known to be associated with cardiovascular development (31). Moreover, DNA methylation of the glucocorticoid receptor gene promoter is an epigenetic mechanism that can lead to abnormal devel- 
A

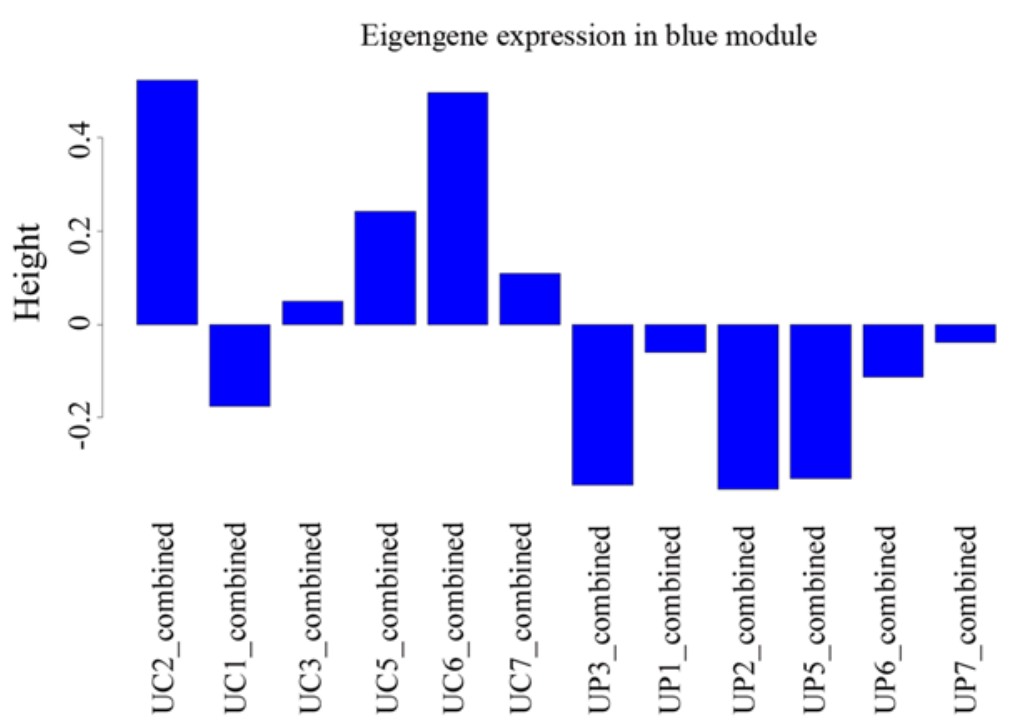

B

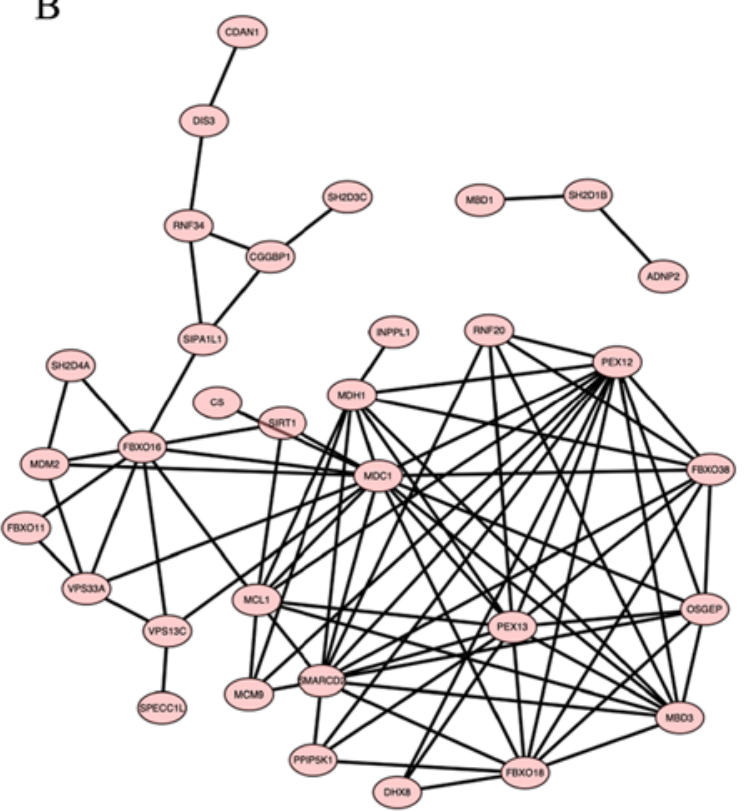

Figure 5. Function analysis of blue module. (A) Eigengene profiles of the blue module. As a result, blue module was expressed higher in healthy pregnant females compared with those who carried pulmonary stenosis infants. (B) Visualization of hub genes in the blue module. UC, samples from the normal group; UP, samples from the PS group.

opment of the fetal heart (32). MicroRNA (miR)-29c-3p overexpression in the serum of pregnant females inhibited embryonic P19 cell proliferation, promoted cell apoptosis and differentiation and is associated with fetal CHD (33). Altogether, these studies suggested that maternal epigenetic changes may play a pivotal role in heart development and pathogenesis of fetal PS.

Glucocorticoid signaling plays an important role in cardiac physiology (34). The effects of glucocorticoids are mediated classically by the glucocorticoid receptor (35). Previous studies indicated that the glucocorticoid receptor in cardiomyocytes is critical for normal development and function of the heart (36-38). Maternal hypoxia caused a significant increase of global methylation in the fetal heart, which was sustained in 4-week-old human infants (30). Maternal hypoxia induced miR-210 production via hypoxia inducible factor- $1 \alpha$, which reduced the expression of glucocorticoid receptors in the fetal heart, and led to cardiomyocyte death (39). In the present study, the expression of the glucocorticoid receptor signaling pathway was reduced in the EVs of pregnant women carrying fetuses with PS offspring.

The results of the present study suggested that EVLRs may be used for the diagnosis of fetal PS. Hub genes involved in 'regulation of glucose transport', 'glucose transport', 'ATP-dependent chromatin remodeling', 'histone deacetylation', 'histone H3-K4 methylation', 'DNA methylation', 'apoptotic signaling pathway', and 'glucocorticoid receptor signaling pathway' were identified, which could represent potential biomarkers for PS. The use of EVLR as a diagnostic would be non-invasive to the fetus. Moreover, hub genes could be easily identified by reverse transcription-quantitative PCR or targeted analysis sequencing from the perinatal blood of pregnant females. Similar to cell-free RNA tests, the latter is a non-invasive blood test for fetal development
(40). However, WGCNA can only provide a cluster of hub genes rather than specific genes related to disease. Thus, the hub genes identified in the present study require further individual validation. Additionally, the cohort enrolled in the present study was relatively small, and further extensive validation is still needed to confirm the present results.

In summary, a profile of EVLR was established for healthy pregnant females and pregnant females with PS, and a gene co-expression network was constructed to predict a cluster of candidate genes involved in the diagnosis of fetal PS. The present findings may provide a theoretical basis for novel, non-invasive detection methods of aberrant fetal heart development.

\section{Acknowledgements}

The authors would like to thank Mr Xuemin Yang (Guangzhou Epibiotek Co., Ltd.) for assisting in sequencing and bioinformatics analysis.

\section{Funding}

The present study was supported by The Natural Science Foundation ofGuangdong(grantno.2018A0303130266),National Key R\&D Program of China (grant no. 2018YFC1002600), the Scientific and Technological Projects of Guangdong (grant nos. 2017A070701013 and 2017B090904034) and the Science and Technological Program of Guangzhou (grant no. 201704020126).

\section{Availability of data and materials}

The datasets used and/or analyzed during the current study are available from the corresponding author on reasonable request. 


\section{Authors' contributions}

ZH, XiaohL, JZ and JimC conceived the study. ZH, MT, FH and XiaohL designed the method. FH provided samples. $\mathrm{ZH}$, XiaohL, MQ, JinC, YO, XiaoqL, CZ and HY prepared the software and performed data analysis. $\mathrm{ZH}$ wrote the original draft. XiaohL reviewed and edited the draft. JZ and JimC supervised and administrated the project. XiaohL, JZ and JimC provided funding. All authors read and approved the final manuscript.

\section{Ethics approval and consent to participate}

The present study was approved by The Ethics Committee of Guangdong Provincial People's Hospital and conducted in accordance with the Declaration of Helsinki. All participants gave their informed oral consent for participation in the study.

\section{Patient consent for publication}

Not applicable.

\section{Competing interests}

The authors declare that they have no competing interests.

\section{References}

1. Liu Y, Chen S, Zühlke L, Black GC, Choy MK, Li N and Keavney BD: Global birth prevalence of congenital heart defects 1970-2017: Updated systematic review and meta-analysis of 260 studies. Int J Epidemiol 48: 455-463, 2019.

2. Cuypers JA, Witsenburg M, van der Linde D and Roos-Hesselink JW: Pulmonary stenosis: Update on diagnosis and therapeutic options. Heart 99: 339-347, 2013.

3. Patel AB, Ratnayaka K and Bergersen L: A Review: Percutaneous pulmonary artery stenosis therapy: state-of-the-art and look to the future. Cardiol Young 29: 93-99, 2019.

4. Webber DM, MacLeod SL, Bamshad MJ, Shaw GM, Finnell RH, Shete SS, Witte JS, Erickson SW, Murphy LD and Hobbs C: Developments in our understanding of the genetic basis of birth defects. Birth Defects Res A Clin Mol Teratol 103: 680-691, 2015.

5. Bahado-Singh RO, Zaffra R, Albayarak S, Chelliah A, Bolinjkar R, Turkoglu $\mathrm{O}$ and Radhakrishna U: Epigenetic markers for newborn congenital heart defect (CHD). J Matern Fetal Neonatal Med 29: 1881-1887, 2016.

6. van Niel G, D'Angelo G and Raposo G: Shedding light on the cell biology of extracellular vesicles. Nat Rev Mol Cell Biol 19 213-228, 2018.

7. Shah R, Patel T and Freedman JE: Circulating extracellular vesicles in human disease. N Engl J Med 379: 958-966, 2018.

8. Tong M, Kleffmann T, Pradhan S, Johansson CL, DeSousa J, Stone PR, James JL, Chen Q and Chamley LW: Proteomic characterization of macro-, micro- and nano-extracellular vesicles derived from the same first trimester placenta: Relevance for feto-maternal communication. Hum Reprod 31: 687-699, 2016

9. Adam S, Elfeky O, Kinhal V, Dutta S, Lai A, Jayabalan N, Nuzhat Z, Palma C, Rice GE and Salomon C: Review: Fetal-maternal communication via extracellular vesicles Implications for complications of pregnancies. Placenta 54 83-88, 2017.

10. Colombo M, Raposo G and Théry C: Biogenesis, secretion, and intercellular interactions of exosomes and other extracellular vesicles. Annu Rev Cell Dev Biol 30: 255-289, 2014.

11. Jin J and Menon R: Placental exosomes: A proxy to understand pregnancy complications. Am J Reprod Immunol 79: e12788, 2018.

12. Zhou R, Chen KK, Zhang J, Xiao B, Huang Z, Ju C, Sun J, Zhang F, Lv XB and Huang G: The decade of exosomal long RNA species: An emerging cancer antagonist. Mol Cancer 17: $75,2018$.
13. Del Re M, Biasco E, Crucitta S, Derosa L, Rofi E, Orlandini C, Miccoli M, Galli L, Falcone A, Jenster GW, et al: The detection of androgen receptor splice variant 7 in plasma-derived exosomal RNA strongly predicts resistance to hormonal therapy in metastatic prostate cancer patients. Eur Urol 71: 680-687, 2017.

14. Li Y, Zhao J, Yu S, Wang Z, He X, Su Y, Guo T, Sheng H, Chen J, Zheng Q, et al: Extracellular vesicles long RNA sequencing reveals abundant mRNA, circRNA, and lncRNA in human blood as potential biomarkers for cancer diagnosis. Clin Chem 65: 798-808, 2019.

15. Bolger AM, Lohse M and Usadel B: Trimmomatic: A flexible trimmer for Illumina sequence data. Bioinformatics 30: 2114-2120, 2014.

16. Kim D, Langmead B and Salzberg SL: HISAT: A fast spliced aligner with low memory requirements. Nat Methods 12: 357-360, 2015.

17. Langfelder P, Horvath $\mathrm{S}$ and Fast R: Fast R functions for robust correlations and hierarchical clustering. J Stat Softw 46: i11, 2012.

18. Langfelder P and Horvath S: WGCNA: An R package for weighted correlation network analysis. BMC Bioinformatics 9: $559,2008$.

19. Shi Z, Derow CK and Zhang B: Co-expression module analysis reveals biological processes, genomic gain, and regulatory mechanisms associated with breast cancer progression. BMC Syst Biol 4: 74, 2010.

20. Liu X, Hu AX, Zhao JL and Chen FL: Identification of key gene modules in human osteosarcoma by co-expression analysis weighted gene co-expression network analysis (WGCNA). J Cell Biochem 118: 3953-3959, 2017.

21. Deng J, Kong W, Mou X, Wang S and Zeng W: Identifying novel candidate biomarkers of RCC based on WGCNA analysis. Per Med 15: 381-394, 2018.

22. Maertens A, Tran V, Kleensang A and Hartung T: Weighted Gene correlation network analysis (WGCNA) Reveals novel transcription factors associated with Bisphenol a dose-response. Front Genet 9: 508, 2018

23. Xi X, Chu Y, Liu N, Wang Q, Yin Z, Lu Y and Chen Y: Joint bioinformatics analysis of underlying potential functions of hsa-let-7b-5p and core genes in human glioma. J Transl Med 17: 129, 2019.

24. Cypryk K, Bartyzel L, Zurawska-Klis M, Mlynarski W, Szadkowska A, Wilczynski J, Nowakowska D, Wozniak LA and Fendler W: Continuous glucose monitoring in type 1 diabetes pregnancy shows that fetal heart rate correlates with maternal glycemia. Diabetes Technol Ther 17: 619-624, 2015.

25. Øyen N, Diaz LJ, Leirgul E, Boyd HA, Priest J, Mathiesen ER, Quertermous T, Wohlfahrt J and Melbye M: Prepregnancy diabetes and offspring risk of congenital heart disease: A nationwide cohort study. Circulation 133: 2243-2253, 2016.

26. Hoang TT, Marengo LK, Mitchell LE, Canfield MA and Agopian AJ: Original findings and updated meta-analysis for the association between maternal diabetes and risk for congenital heart disease phenotypes. Am J Epidemiol 186: 118-128, 2017.

27. Helle EIT, Biegley P, Knowles JW, Leader JB, Pendergrass S, Yang W, Reaven GR, Shaw GM, Ritchie M and Priest JR: First trimester plasma glucose values in women without diabetes are associated with risk for congenital heart disease in offspring. J Pediatr 195: 275-278, 2018.

28. Priest JR, Yang W, Reaven G, Knowles JW and Shaw GM: Maternal Midpregnancy glucose levels and risk of congenital heart disease in offspring. JAMA Pediatr 169: 1112-1116, 2015.

29. Moore-Morris T, van Vliet PP, Andelfinger G and Puceat M: Role of epigenetics in cardiac development and congenital diseases. Physiol Rev 98: 2453-2475, 2018.

30. Radhakrishna U,Albayrak S,Zafra R,Baraa A, Vishweswaraiah S, Veerappa AM, Mahishi D, Saiyed N, Mishra NK, Guda C, et al: Placental epigenetics for evaluation of fetal congenital heart defects: Ventricular Septal Defect (VSD). PLoS One 14: e0200229, 2019.

31. Muntean I, Togănel R and Benedek T: Genetics of congenital heart disease: Past and Present. Biochem Genet 55: 105-123, 2017.

32. Song $\mathrm{R}, \mathrm{Hu} \mathrm{XQ}$ and Zhang L: Glucocorticoids and programming of the microenvironment in heart. J Endocrinol 242: T121-T133, 2019.

33. Chen T, Li SJ, Chen B, Huang Q, Kong XY, Shen C, Gu HT and Wang XW: Akt3 is a target of miR-29c-3p and serves an important function in the pathogenesis of congenital heart disease. Int J Mol Med 43: 980-992, 2019.

34. Fowden AL and Forhead AJ: Glucocorticoids as regulatory signals during intrauterine development. Exp Physiol 100: $1477-1487,2015$. 
35. Weikum ER, Knuesel MT, Ortlund EA and Yamamoto KR: Glucocorticoid receptor control of transcription: Precision and plasticity via allostery. Nat Rev Mol Cell Biol 18: 159-174, 2017.

36. Rog-Zielinska EA, Thomson A, Kenyon CJ, Brownstein DG, Moran CM, Szumska D, Michailidou Z, Richardson J, Owen E, Watt A, et al: Glucocorticoid receptor is required for foetal heart maturation. Hum Mol Genet 22: 3269-3282, 2013.

37. Rog-Zielinska EA, Craig MA, Manning JR, Richardson RV Gowans GJ, Dunbar DR, Gharbi K, Kenyon CJ, Holmes MC, Hardie DG, et al: Glucocorticoids promote structural and functional maturation of foetal cardiomyocytes: A role for PGC-1 $\alpha$. Cell Death Differ 22: 1106-1116, 2015.
38. Oakley RH and Cidlowski JA: Glucocorticoid signaling in the heart: A cardiomyocyte perspective. J Steroid Biochem Mol Biol 153: 27-34, 2015

39. Martinez SR, Ma Q, Dasgupta C, Meng X and Zhang L: MicroRNA-210 suppresses glucocorticoid receptor expression in response to hypoxia in fetal rat cardiomyocytes. Oncotarget 8 : 80249-80264, 2017.

40. Ngo TTM, Moufarrej MN, Rasmussen MH, Camunas-Soler J, Pan W, Okamoto J, Neff NF, Liu K, Wong RJ, Downes K, et al: Noninvasive blood tests for fetal development predict gestational age and preterm delivery. Science 360: 1133-1136, 2018 